\title{
THE CORRELATION OF SOLAR RADIO BURSTS WITH MAGNETIC ACTIVITY AND COSMIC RAYS
}

\author{
A. R. THOMPSON \\ Harvard Radio Astronomy Station, Fort Davis, Texas, U.S.A.
}

The sweep-frequency equipment at the Harvard Radio Astronomy Station, Fort Davis, Texas, has now been running continuously since 1956 September, recording solar radio activity in the frequency range from 100 to $580 \mathrm{Mc} / \mathrm{s}$. The following contribution describes preliminary investigations of the correlation of the radio data with solar corpuscular emissions. This work was initiated to examine the well-known suggestions that the origins of the type II and type III radio bursts are associated with the ejection of auroral particles and cosmic rays respectively.

In the type II, or slow-drift, bursts the rate of change of frequency indicates an origin in a region of the solar atmosphere which moves outward at a velocity of the order of $1500 \mathrm{~km} / \mathrm{second}$. This velocity corresponds to a time of travel between the sun and the earth of 28 hours, and suggests the auroral association. In the following investigation the values of $K_{p}$, the index of magnetic activity, have been taken as an index of auroral emission, and records of 77 type II bursts that occurred between 1956 October 1 and 1958 April 30 have been used. Twenty-six of these bursts were recorded on the sweep-frequency equipment at Sydney, Australia, and 51 at Fort Davis.

The three hourly $K_{p}$ values have been taken for each burst over a period commencing two days before the burst, and ending seven days after it. These values have then been averaged over the sample of 77 bursts, and appear as the ordinate of the superposed epoch diagram in Fig. 1. The curve shows clearly an increase in the magnetic activity, reaching a peak centered on a

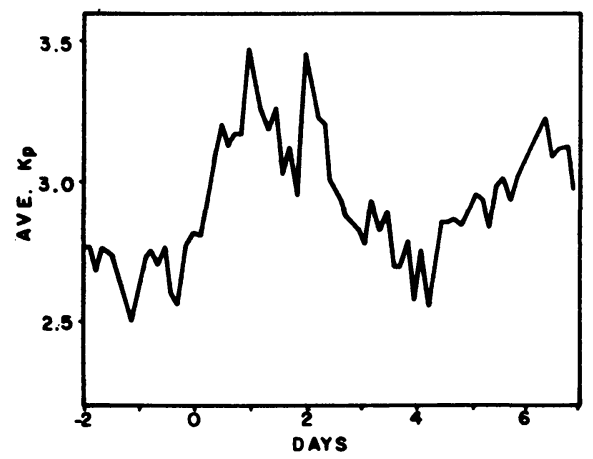

Fig. 1. Superposed epoch diagram showing variation of $K p$ for 77 type II bursts. time approximately 1.5 days after the onset of the burst. A second and smaller peak occurs about 4.5 days after the first one, and is probably a result of the time distribution of the type II bursts; 24 out of the 77 used in the analysis were followed by a second burst within a period of 3 to 5 days.

Where optical data are available 90 per cent of the type II bursts are associated with solar flares. It is therefore of interest to compare the results of Fig. 1 with those obtained 
by Dodson and Hedeman [1] in an investigation of the relationship between flares and the $K_{p}$ index. Dodson and Hedeman found that a peak in magnetic activity occurs about 2.5 days after a flare, and that the flares most strongly associated with a magnetic disturbance are those accompanied by a major early burst, i.e. an intense radio burst preceding the maximum of the flare by a few minutes. The radio data used by Dodson and Hedeman were mainly obtained from singlefrequency equipment, and it is therefore not certain to what spectral classification the major early bursts belong. However, if they are not of spectral type II, they probably corre-

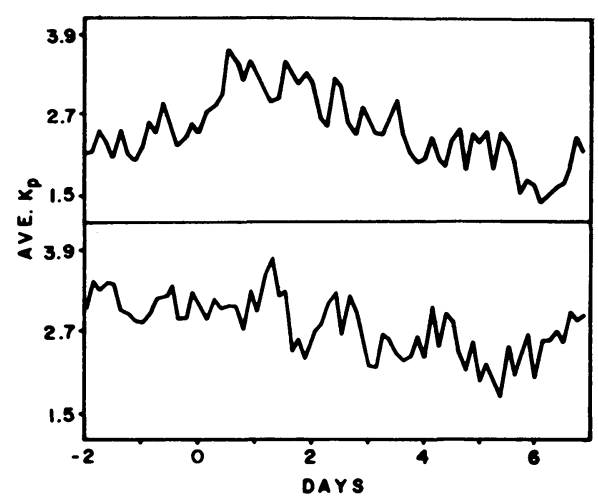

FIG. 2. Superposed epoch diagrams showing variation of $K p$, upper curve for eight type II bursts that commenced before the maxima of the associated flares, and lower curve for eight type II bursts that commenced after the maxima of the associated flares. spond to the intense groups of type III bursts that often precede the type II bursts. About 66 per cent of the type II bursts recorded at Fort Davis are accompanied by such a type III group, but the presence of this group appears to have no effect upon the correlation with magnetic activity. This confirms that the radio bursts of geomagnetic importance are those of spectral type II.

A study of the flare correlation of the 77 type II bursts in Fig. 1 shows that after eliminating those cases in which there is no flare correlation, ambiguous flare correlation, or incomplete information on the time of flare maximum, 16 cases remain. In these, 8 of the bursts commence before the maximum of the flare, and 8 afterwards. Superposed epoch diagrams of $K_{p}$ variation for these two sets of 8 bursts are shown in Fig. 2. The bursts preceding flare maximum show an increase in the average $K_{p}$ values of about twice that in Fig. 1, and those following flare maximum show no clearly pronounced increase at all. This suggests that about 50 per cent of flarecorrelated type II bursts commence before flare maximum, and that they are the ones which are followed by a magnetic disturbance. This is in agreement with the work of Dodson and Hedeman, but must be regarded as merely a tentative result. The number of bursts used in each curve of Fig. 2 is much too small to permit any firm conclusions to be drawn.

The second part of this investigation concerns the relationship between solar radio bursts and cosmic rays. In the type III or fast-drift bursts the rate of frequency change is much higher than that of the type II bursts, and corresponds to a source moving outward through the solar corona with a velocity of the order of $50,000 \mathrm{~km} / \mathrm{second}$. Wild, Roberts, and Murray [2] have suggested that the origin of type III bursts is associated with the emission of solar cosmic rays.

In the following investigation the method that has been used is to compare, 
for a period of several months, the daily average figures of radio emission and cosmic ray count. Fig. 3 shows the results for the period 1957 August through 1958 February. Radio-emission curves for both type III bursts and noise storm activity are shown, and in each case the ordinate is proportional to the energy received during each daily period from sunrise to sunset. The cosmic ray intensity was obtained from the hourly figures of the neutron monitor pile at Climax, Colorado.

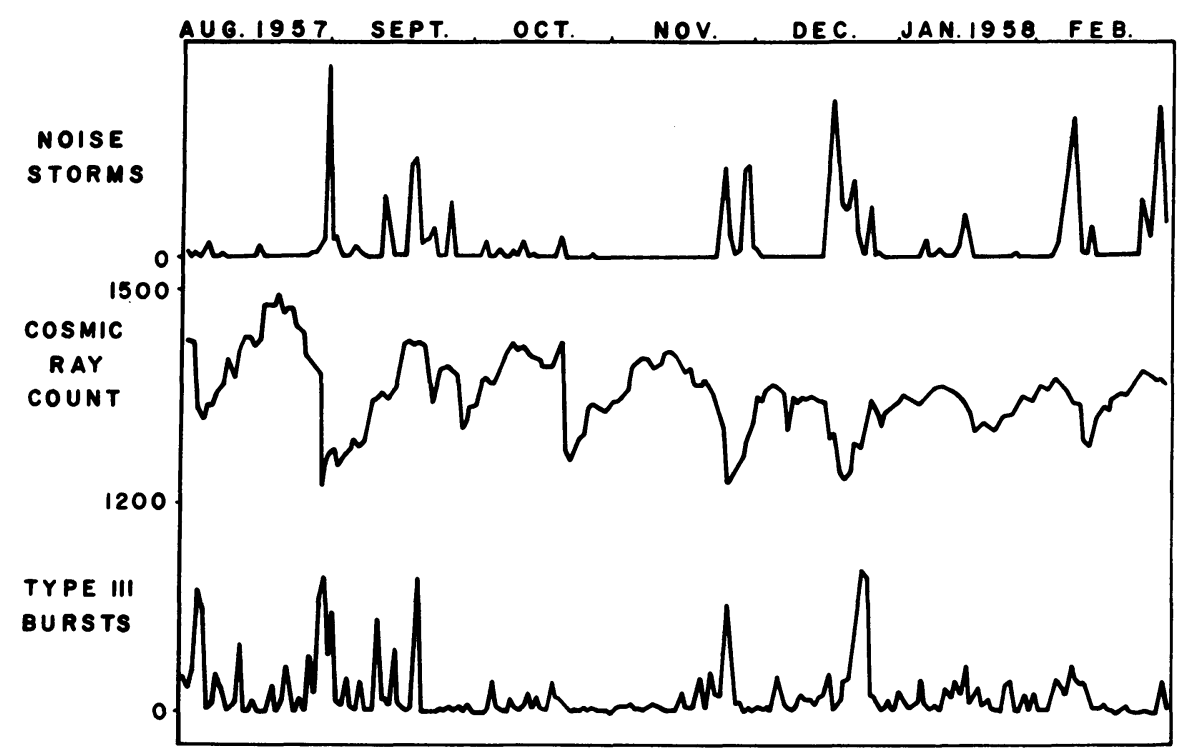

FIG. 3. Daily averaged values of cosmic ray intensity compared with noise storm and type III activity.

A comparison of the curves in Fig. 3 shows no evidence of increase in cosmic ray intensity corresponding to periods of type III activity. Several periods of intense radio emission correspond rather to decreases in cosmic ray intensity. A similar effect has been noted by Tandberg-Hanssen [3], for single-frequency radio observations below $200 \mathrm{Mc} / \mathrm{s}$. Tandberg-Hanssen suggests that some decrease of cosmic ray intensity may be due to an ionized cloud in the solar atmosphere, inhibiting the emission of a solar component of cosmic rays, and generating radio noise.

In conclusion, it appears from the above investigations that there is evidence to support theories relating type II bursts to auroral emission. The attempt to obtain similar evidence for type III bursts and cosmic rays has so far been unsuccessful; some periods of intense solar radio emission appear to be related rather to decreases in cosmic ray intensity.

Other participants in this work were Dr. Alan Maxwell of the Harvard Radio Astronomy Station, and Mr. Gordon Garmire, and Mr. Peter Stone of Harvard University. The Australian sweep-frequency data were kindly supplied by Mr. J. P. Wild of C.S.I.R.O., Sydney, and the cosmic ray data by 
Professor J. A. Simpson of the Fermi Institute for Nuclear Studies, University of Chicago. The work at the Harvard Radio Astronomy Station at Fort Davis is supported financially by the Air Force Cambridge Research Center.

\section{REFERENCES}

[1] Dodson, H. W., and Hedeman, E. R. J. Geophys. Res. 63, 77, 1958.

[2] Wild, J. P., Roberts, J. A., and Murray, J. D. Nature, 173, 532, 1954.

[3] Tandberg-Hanssen, E. Unpublished report, 1956. 